CLINICAL STUDY

\title{
Well-being, mood and calcium homeostasis in patients with hypoparathyroidism receiving standard treatment with calcium and vitamin $\mathbf{D}$
}

\author{
Wiebke Arlt, Christian Fremerey, Frank Callies, Martin Reincke, Peter Schneider ${ }^{1}$, Wolfgang Timmermann ${ }^{2}$ and \\ Bruno Allolio \\ Department of Medicine, Endocrine and Diabetes Unit, ${ }^{1}$ Department of Nuclear Medicine and ${ }^{2}$ Department of Surgery, University of Wuerzburg, Germany \\ (Correspondence should be addressed to W Arlt, Department of Medicine, Endocrine and Diabetes Unit, University of Wuerzburg, \\ Josef-Schneider-Strasse 2, 97080 Wuerzburg, Germany; Email: w.arlt@medizin.uni-wuerzburg.de)
}

\begin{abstract}
Objective: Standard treatment in hypoparathyroidism consists of calcium and vitamin D (or vitamin D analogs) but does not employ replacement of the actual missing hormone. Only few studies have evaluated the efficacy of calcium/vitamin D treatment in hypoparathyroidism; the impact of chronic hypoparathyroid disease on well-being has not been investigated previously.

Design: Cross-sectional, controlled study in 25 unselected women with postsurgical hypoparathyroidism since $6.4 \pm 8.0$ years (S.D.) on stable treatment with calcium and vitamin D (or analogs) and in 25 controls with a history of thyroid surgery but intact parathyroid function, who were matched for sex, age and time since surgery.

Methods: Assessment of well-being and mood using validated questionnaires (the revised version Symptom Checklist 90 (SCL-90-R); the Giessen Complaint List (GBB-24); and the von Zerssen Symptom List (B-L Zerssen)), serum and urinary calcium/phosphorus homeostasis, and in the hypoparathyroid patients also screening for secondary disease by kidney ultrasound, ophthalmological split lamp examination, and measurement of bone mineral density.

Results: Serum calcium was in the accepted therapeutic range in the majority of hypoparathyroid patients. However, calcium/phosphorus homeostasis as a whole was clearly non-physiological. Nephrolithiasis was detected in 2 and cataracts in 11 of 25 hypoparathyroid patients. As compared with controls, hypoparathyroid patients had significantly higher global complaint scores in GBB-24 $(P=0.036)$, B-L Zerssen $(P=0.002)$ and SCL-90-R $(P=0.020)$ with predominant increases in the subscale scores for anxiety, phobic anxiety and their physical equivalents.

Conclusions: Current standard treatment in hypoparathyroidism is not only associated with an altered calcium/phosphorus homeostasis but also fails to restore well-being in these patients. Future studies need to address the impact of more physiological treatment options like parathyroid hormone $(1-34)$ or parathyroid transplantation on well-being and mood in these patients.
\end{abstract}

European Journal of Endocrinology 146 215-222

\section{Introduction}

The reported incidence of permanent hypoparathyroidism following thyroid surgery varies between 0.5 and $4 \%$ depending on the extent of operation and the surgical technique as well as on the experience of the surgeon (1-3). In countries with a high prevalence of goiter $(4,5) 500$ thyroid surgeries per one million inhabitants are performed every year (1). Thus, in Germany an annual number of 500-1000 new patients with postsurgical hypoparathyroidism (PSHP) is to be expected.

The widely accepted standard treatment for hypoparathyroidism consists of supplementation with calcium and vitamin D, vitamin D metabolites or vitamin D analogs (6-9), but does not employ replacement of the actual missing hormone, parathyroid hormone (PTH). This is a major difference from the more physiological replacement concepts used in the treatment of other hormonal deficiencies (e.g. adrenal insufficiency or hypothyroidism).

There is no doubt that calcium and vitamin D administration ends life-threatening hypocalcemia and debilitating tetanic symptoms in hypoparathyroid patients. However, calcium/vitamin D does not restore the physiological calcium/phosphorus homeostasis (10). Furthermore, evaluation of the clinical efficacy of calcium/ vitamin D treatment in chronic hypoparathyroidism 
has been largely neglected and well-being or mood in these patients has not been studied. Thus we performed a cross-sectional study in patients with hypoparathyroidism analyzing calcium homeostasis as well as well-being and mood, comparing the results with those obtained in sex- and age-matched controls with a history of thyroid surgery but intact parathyroid function.

\section{Methods}

\section{Patients}

We contacted by mail all outpatients with the established diagnosis of hypoparathyroidism currently registered at the Endocrine Ambulatory Unit of the University Hospital Wuerzburg $(n=36)$ and invited them to participate in the study. Twenty-nine of 36 patients were interested in participation (one man and 25 women with PSHP, two women with pseudohypoparathyroidism type Ia, and one woman with idiopathic hypoparathyroidism). In order to recruit a homogenous group, we included only the 25 women

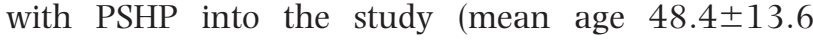
years (s.D.) (range 24-66 years); duration of disease, median 3 years, range $0.5-38$ years). They suffered from PSHP following subtotal thyroidectomy for goiter $(n=23)$ or parathyroid surgery for hyperparathyroidism $(n=2)$. All patients received long-standing, stable treatment with calcium $(0.5-3.0 \mathrm{~g} /$ day $)$ and oral vitamin D, vitamin D metabolites or analogs (cholecalciferol 0.625-0.25 mg/day $(n=2), \alpha$-calcidol $1-2 \mu \mathrm{g} /$ day $(n=3)$, calcitriol $0.25-1.25 \mu \mathrm{g} /$ day $(n=13)$, or dihydrotachysterol $0.25-0.50 \mathrm{mg} /$ day $(n=8)$. Seven of 25 patients received additional magnesium replacement.

For each hypoparathyroid patient, two potential control subjects with a history of subtotal thyroidectomy for goiter but intact parathyroid function were selected from the register files of the Department of Surgery, University of Wuerzburg. All selected control subjects were matched for sex, age and time since surgery. The first of the two potential controls agreeing to participate in the study was included. Thus, it was possible to compare the 25 patients with PSHP with a control group of 25 women with normal parathyroid function (mean age 49.5 \pm 13.2 years, range 24-67 years).

Fifteen female volunteers as well as 15 of the female PSHP patients were postmenopausal; two and three respectively received estrogen/progestin replacement. Twenty of the 25 PSHP patients and 22 of the 25 controls took thyroxine replacement $(75-150 \mu \mathrm{g} /$ day $)$. In all subjects thyrotropin measurements reflected euthyroid function. At the time of testing, none of the participants suffered from any other chronic or acute disease. Further exclusion criteria were history of past psychiatric disease and past or current use of psychotropic drugs.
Prior to initiation of the study, the Ethics Committee of the University of Wuerzburg approved the study protocol, and all patients gave written informed consent.

\section{Evaluation}

All participants reported to the ambulatory unit during the morning having taken their usual medication (calcium, vitamin D or analogs, and thyroxine). After history taking and clinical examination, psychometric evaluation was carried out starting with a detailed and standardized instruction in how to complete the three different questionnaires. Afterwards, blood was drawn from all participants for determination of parameters of calcium homeostasis and renal function. In the hypoparathyroid patients this was followed by kidney ultrasound, measurement of bone mineral density (BMD) and ophthalmological split lamp examination. Patients and controls were instructed how to collect a $24 \mathrm{~h}$ urine and asked to bring it with them on one of the future appointments. Six weeks after the initial psychometric evaluation, an identical set of questionnaires was mailed to all participants, who were asked to send it back after completion.

\section{Measurements}

Psychometric evaluation of patients and controls was performed using three validated questionnaires, the revised version Symptom Checklist 90 (SCL-90-R), the von Zerssen Symptom List (B-L Zerssen), and the short form of the Giessen Complaint List (GBB-24). With the help of these questionnaires mood and well-being were assessed, the latter mainly as the subjective health status. All questionnaires are self-report or self-assessment questionnaires, which do not require the active participation of an interviewer or investigator but are presented as a self-explanatory written multiple-choice test.

The SCL-90-R (11) is a multidimensional self-report symptom inventory consisting of 90 items measuring a global severity index (GSI) and nine psychological dimensions (or subscales): somatization, obsessivecompulsive traits, interpersonal sensitivity, depression, anxiety, hostility, phobic anxiety, paranoid ideation, and psychotic tendencies. The responders are asked to rate items on five-step scales from 'I completely disagree' (or 'not at all') to 'I completely agree' (or 'extremely') taking into account their feelings during the preceding 7 days including the day of the testing. For calculations, the items on the scales are transformed into values from 0 (not at all) to 4 (extremely) and subscale scores are calculated by adding up the respective ratings and dividing by the number of items. The GSI is calculated from the sum of all ratings divided by the number of rated items. The average scores from the SCL-90-R can be additionally converted to standard T-scores (ranging from a minimum of 30 to 
a maximum of 80) by referring to the appropriate female population-based norm tables provided by the test manual based on a reference sample of 18- to 60 -year-old healthy women $(n=501)(12)$. A T-score of 50 represents the mean T-score of the respective normal population and the T-score range from 40 to 60 represents the normal range (as defined by the mean \pm S.D.). T-scores $>55$ represent an already significant impairment and a T-score $>60$ represents a pathological result.

The B-L Zerssen (13) is a general well-being scale consisting of two similar questionnaires (B-L and B-L') with 24 items each. The items are rated on a fourstep scale from 0 to 3 and scores are subsequently calculated by summing the item ratings. The global score is calculated from the mean of both scores and then further converted to a T-score and a percentile value by referring to the norm tables for females deriving from a population-based reference sample of 20- to 64-year-old women $(n=988)$ (13).

The short form of the GBB-24 (14) consists of 24 items defining four subscales (exhaustion tendency, gastric symptoms, pain in the limbs, and heart complaints) each including six items with ratings from 0 to 4. Additionally, a global score of discomfort (GSD) is calculated by adding up the four subscale scores. The GSD and the four subscale scores can be further converted to quartile values by referring to age-dependent norm tables for females provided by the test manual (15) based on a reference sample of healthy 18 - to 60 -year-old women $(n=732)$ stratified by age decade.

The higher the score or subscale score in any of the three psychometrical instruments, the greater the impairment of well-being as assessed by the respective questionnaire.

In patients and controls, biochemical measurements included calcium and phosphorus in serum and in $24 \mathrm{~h}$ urine, as well as serum renal function parameters (creatinine, urea, albumin and total protein). These measurements were carried out using enzymatic colorimetric standard methods.

The hypoparathyroid patients underwent additional tests including ophthalmological split lamp examination, imaging of the kidneys by abdominal ultrasound and measurement of BMD.

BMD measurements were carried out at the lumbar spine (L1-L4) and at the hip (neck and trochanter) by dual-energy X-ray absorptiometry (DXA) using a Lunar-DPX-Scanner (Lunar Corporation, Madison, WI, USA). Additionally, volumetric total and trabecular density were measured at the non-dominant distal radius by peripheral quantitative computed tomography (pQCT) using an XCT900 scanner (Stratec Medizintechnik, Pforzheim, Germany). Daily quality control was assured in both scanners, using the European Spine Phantom and the European Forearm Phantom. The results of lumbar spine and hip BMD were compared with population-based European reference ranges $(16,17)$. Results at the distal radius were compared with a population-based German reference sample (18).

\section{Statistical analysis}

Data are presented as means \pm S.D. Comparison of data was performed by $t$-test for unpaired samples, and if parameters were not normally distributed, by the non-parametric Mann-Whitney test. In addition, linear regression analyses were performed. Significance was defined as $P<0.05$.

Although the psychometric test instruments have shown good re-test reliability $(12,13,15)$, we employed two separate psychometric evaluation sessions; once during the clinic visit and once at home 6 weeks later. From the results of the two responses the average was taken and used for further statistical analysis.

The individual BMD results from both DXA and pQCT measurements were transformed into z-scores using European and German reference databases (16-18). The data of the two patients with a history of previous hyperparathyroidism were excluded from statistical analysis. Four patients did not participate in BMD measurements, hence we were able to analyze BMD data from 19 hypoparathyroid patients.

\section{Results}

\section{Calcium and phosphorus homeostasis}

Serum calcium values in 18 of 25 hypoparathyroid patient sera were within the accepted therapeutic range (i.e. the lower normal range, 2.00$2.35 \mathrm{mmol} / \mathrm{l}$ ), in the others serum calcium was either within the higher normal range $(2.36-2.70 \mathrm{mmol} / \mathrm{l}$, $n=4)$ or slightly below the normal range $(n=3)$. Serum phosphorus was within the normal range $(0.8-1.5 \mathrm{mmol} / \mathrm{l})$ in 20 of 25 hypoparathyroid patients. However, in comparison with controls, patients with hypoparathyroidism had a significantly lower mean serum calcium (patients vs controls 2.15士 0.21 vs $2.27 \pm 0.09 \mathrm{mmol} / \mathrm{l}, P=0.016$ ) and a significantly higher mean serum phosphorus concentration (1.32 \pm 0.22 vs $1.03 \pm 0.13 \mathrm{mmol} / \mathrm{l}, P<0.0001)$. In the hypoparathyroid patients, there was a negative correlation between serum calcium and serum phosphorus $(r=-0.42, P=0.035)$ (Fig. 1A) while there was a positive one in the controls $(r=0.46, P=$ 0.019) (Fig. 1B). In 5 of 22 patients (and in 1 of 23 controls) urinary $24 \mathrm{~h}$ excretion of calcium exceeded the upper limit of the normal range $(3-8 \mathrm{mmol} /$ day $)$. The two groups did not differ concerning $24 \mathrm{~h}$ urinary calcium excretion (patients vs controls 5.51 \pm 4.17 vs $4.08 \pm 2.03 \mathrm{mmol} / 24 \mathrm{~h}, P=0.15)$ or $24 \mathrm{~h}$ phosphorus excretion $(26.1 \pm 8.8$ vs $24.9 \pm 8.7 \mathrm{mmol} / 24 \mathrm{~h}$, 


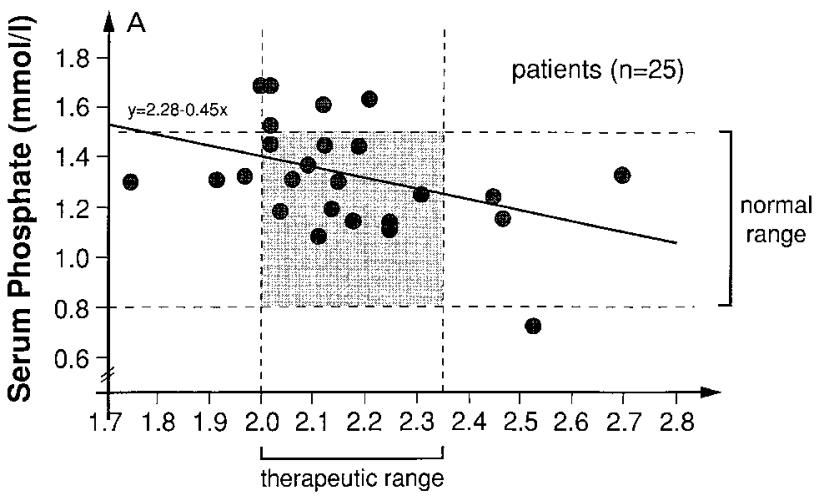

Serum Calcium $(\mathrm{mmol} / \mathrm{l})$

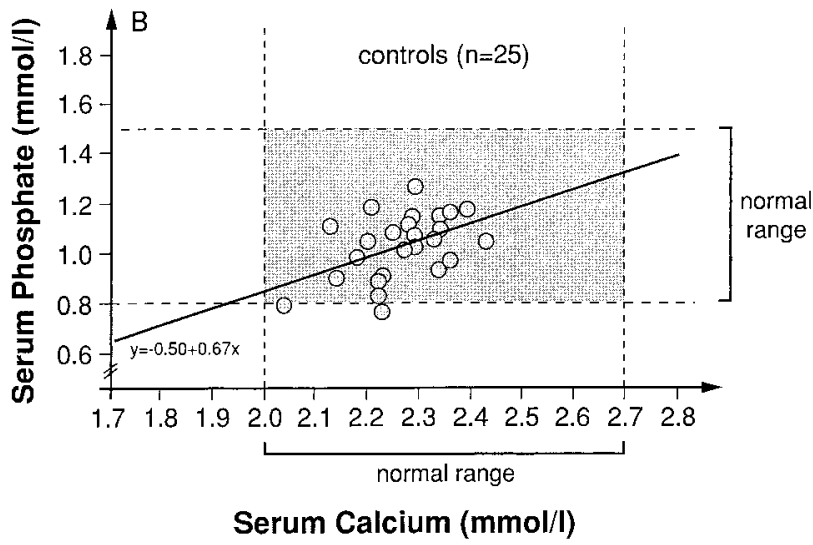

Figure 1 Serum calcium vs serum phosphorus in (A) patients with hypoparathyroidism $(n=25)$ receiving long-standing therapy with oral calcium and vitamin $\mathrm{D}$ preparations $(r=-0.42, P=0.035)$ and in (B) controls $(n=25)$ with intact parathyroid function $(r=0.46, P=0.019)$.

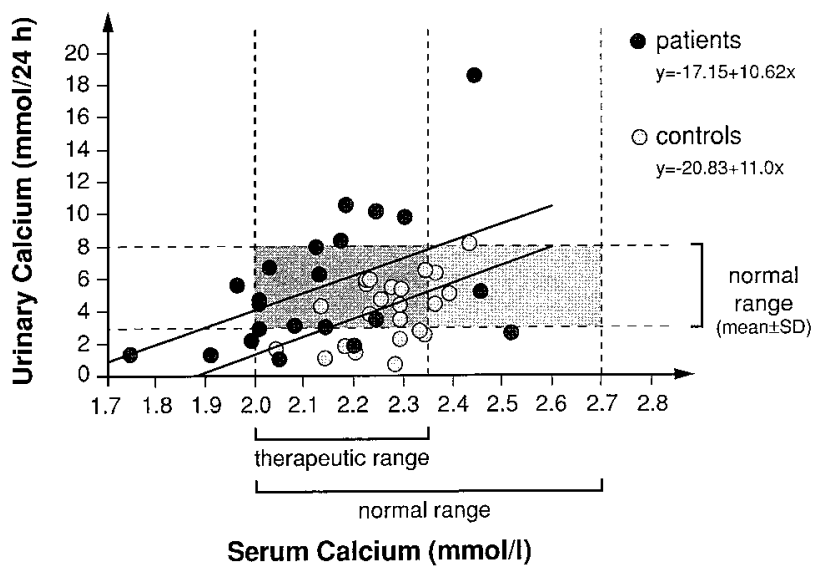

Figure 2 Serum calcium vs $24 \mathrm{~h}$ urinary excretion of calcium in patients with hypoparathyroidism $(n=22)$ receiving long-standing therapy with oral calcium and vitamin D preparations $(r=0.48$, $P=0.024)$ and in controls $(n=23)$ with intact parathyroid function $(r=0.49, P=0.016)$
$P=0.64)$. Urinary calcium excretion and serum calcium correlated positively in both groups (patients, $r=$ $0.48, P=0.024$; controls, $r=0.49, P=0.016$ ), with an upward shift of the regression line in the hypoparathyroid patients (Fig. 2) that illustrates the nonphysiological calcium/phosphorus homeostasis in these patients.

\section{Well-being and mood}

In comparison with controls, hypoparathyroid patients had significantly higher SCL-90-R subscale scores for somatization, depression, anxiety, and phobic anxiety, with differences so pronounced that they even accounted for a significantly higher GSI (Table 1). Test scores taken during the clinic visit did not differ from those taken at home. In the hypoparathyroid patients conversion to standard T-scores revealed pathologically elevated mean T-scores for anxiety, phobic anxiety, somatization and the GSI, while mean T-scores in the controls generally varied around the mean of the normal range (Table 1). Looking at individual T-scores for the GSI revealed that 12 of 25 patients but only 6 of 25 controls had a T-score higher than 60 , equivalent to severe impairment of well-being and mood and an additional 5 patients and 3 controls presented with a T-score higher than 55 , representing an already significant impairment of mood.

The results of the GBB-24 further confirmed this pattern. As compared with controls, patients had significantly higher scores for exhaustion tendency, pain in the limbs, heart complaints and the GSD (Table 2). The differences between patients and controls were so pronounced that they even accounted for significantly different quartile values for heart complaints (i.e. palpitations) and the GSD (Table 2).

Consistent with these findings, the global score of the B-L Zerssen was significantly higher in the hypoparathyroid patients (patients vs controls $29.6 \pm 14.0$ vs $18.7 \pm 9.5, P=0.002$ ). According to T-scores (patients vs controls $62.3 \pm 10.6$ vs $53.8 \pm 7.7, P=0.002$ ) and percentiles $(79.7 \pm 22.5$ vs $62.0 \pm 24.2, P=0.009)$, controls ranged near the normal mean while in the patients mean scores exceeded the upper normal limit.

There was no correlation between duration of hypoparathyroidism and global complaint scores (GSI (SCL90-R): $r=0.12, P=0.57$; global score Zerssen: $r=$ $-0.16, \quad P=0.46 ; \quad$ GSD $\quad($ GBB-24): $\quad r=-0.08$, $P=0.72$ ) or one of the SCL-90-R subscale scores (somatization: $r=-0.02, \quad P=0.95$; anxiety: $r=$ $-0.24, P=0.25$; depression: $r=-0.30, P=0.14$; phobic anxiety: $r=-0.11, P=0.60)$.

Similarly, serum calcium in the patients was correlated neither with one of the global complaint scores (GSI (SCL-90-R): $r=0.11, P=0.60$; B-L Zerssen: $r=$ $0.08, P=0.70$; GSD (GBB-24): $r=0.001, P=1.0$ ) nor with one of the SCL-90-R subscale scores (somatization: $r=0.02, P=0.92$; anxiety: $r=-0.08, P=0.70$; 
Table 1 SCL-90-R subscale scores and standard T-scores (means \pm S.D.) in female patients with hypoparathyroidism $(n=25)$ and in sex- and age-matched controls $(n=25)$ with a history of thyroid surgery but intact parathyroid function. Normal range for females according to (12).

\begin{tabular}{|c|c|c|c|c|c|}
\hline SCL-90-R subscales & Score & Patients & Controls & $P$-value & $\begin{array}{c}\text { Female normal range } \\
\quad(\text { means } \pm \text { S.D. })\end{array}$ \\
\hline \multirow[t]{2}{*}{ Somatization } & Score value & $1.02 \pm 0.69$ & $0.65 \pm 0.40$ & 0.023 & $0.41 \pm 0.31$ \\
\hline & T-value & $64.4 \pm 12.2$ & $57.4 \pm 10.2$ & 0.032 & $50 \pm 10$ \\
\hline \multirow[t]{2}{*}{ Obsessive-compulsive traits } & Score value & $0.81 \pm 0.55$ & $0.57 \pm 0.11$ & 0.138 & $0.51 \pm 0.39$ \\
\hline & T-value & $56.6 \pm 11.5$ & $50.1 \pm 14.2$ & 0.082 & $50 \pm 10$ \\
\hline \multirow[t]{2}{*}{ Interpersonal sensitivity } & Score value & $0.60 \pm 0.47$ & $0.43 \pm 0.56$ & 0.244 & $0.47 \pm 0.41$ \\
\hline & T-value & $53.5 \pm 10.6$ & $48.3 \pm 12.5$ & 0.116 & $50 \pm 10$ \\
\hline \multirow[t]{2}{*}{ Depression } & Score value & $0.70 \pm 0.46$ & $0.42 \pm 0.46$ & 0.037 & $0.49 \pm 0.42$ \\
\hline & T-value & $54.7 \pm 10.0$ & $47.2 \pm 11.3$ & 0.016 & $50 \pm 10$ \\
\hline \multirow[t]{2}{*}{ Anxiety } & Score value & $0.76 \pm 0.12$ & $0.33 \pm 0.35$ & 0.003 & $0.35 \pm 0.35$ \\
\hline & T-value & $59.8 \pm 11.1$ & $49.2 \pm 10.4$ & 0.001 & $50 \pm 10$ \\
\hline \multirow[t]{2}{*}{ Hostility } & Score value & $0.44 \pm 0.35$ & $0.18 \pm 0.23$ & 0.004 & $0.38 \pm 0.36$ \\
\hline & T-value & $52.2 \pm 9.5$ & $44.0 \pm 8.3$ & 0.002 & $50 \pm 10$ \\
\hline \multirow[t]{2}{*}{ Phobic anxiety } & Score value & $0.49 \pm 0.59$ & $0.21 \pm 0.43$ & 0.0626 & $0.17 \pm 0.26$ \\
\hline & $\mathrm{T}$-value & $57.9 \pm 12.1$ & $49.9 \pm 10.5$ & 0.017 & $50 \pm 10$ \\
\hline \multirow[t]{2}{*}{ Paranoid ideation } & Score value & $0.54 \pm 0.40$ & $0.41 \pm 0.57$ & 0.357 & $0.38 \pm 0.38$ \\
\hline & T-value & $54.2 \pm 9.6$ & $49.4 \pm 11.5$ & 0.122 & $50 \pm 10$ \\
\hline \multirow[t]{2}{*}{ Psychotic tendencies } & Score value & $0.28 \pm 0.28$ & $0.11 \pm 0.20$ & 0.023 & $0.21 \pm 0.26$ \\
\hline & T-value & $53.2 \pm 9.2$ & $45.4 \pm 8.2$ & 0.003 & $50 \pm 10$ \\
\hline \multirow[t]{2}{*}{ Global severity index (GSI) } & Score value & $0.67 \pm 0.38$ & $0.44 \pm 0.36$ & 0.034 & $0.39 \pm 0.26$ \\
\hline & T-value & $59.1 \pm 11.2$ & $51.2 \pm 11.9$ & 0.020 & $50 \pm 10$ \\
\hline
\end{tabular}

depression: $r=-0.22, P=0.30$; phobic anxiety: $r=$ $-0.19, P=0.38)$. The exclusion of patients with insufficiently controlled hypoparathyroidism (serum calcium less than 2.00 or higher than $2.40 \mathrm{mmol} / \mathrm{l}$ ) did not affect the outcome, e.g. the scale value for the GSI (SCL-90-R) was $0.44 \pm 0.36$ in the controls and $0.67 \pm$ 0.38 in all patients with $0.70 \pm 0.38$ in the well-controlled patients $(n=17)$ and $0.57 \pm 0.37$ for poorly controlled patients $(n=18)$. Thus, poorly controlled patients presented with even less impaired well-being.

\section{Secondary disease}

In all patients and controls, serum parameters of renal function were within the normal range. Abdominal ultrasound detected previously asymptomatic unilateral kidney stones in 2 of 25 hypoparathyroid patients; there was no case of nephrocalcinosis.
Ophthalmological split lamp examination revealed incipient $(n=8)$ and manifest $(n=3)$ cataract in 20 patients examined (localization: unilateral 2 of 11 , bilateral 9 of 11 ; type: nuclear $(n=2)$, cortical $(n=$ $8)$ and subcapsular $(n=1)$. Patients with cataract tended to have a longer duration of hypoparathyroidism than those without $(7.5 \pm 11.0$ vs $4.8 \pm 4.0$ years, $P=0.49)$. Patients with cataract also tended to be older (53.6 \pm 15.3 vs $43.2 \pm 11.5$ years, $P=0.11$ ), although cataracts were also diagnosed in two 24and 25-year-old women with a duration of disease of 5 and 11 years respectively.

\section{BMD}

BMD in the hypoparathyroid patients was significantly higher than in normal persons of similar age, both at the lumbar spine (BMD (L1-L4) $1.28 \pm 0.18 \mathrm{~g} / \mathrm{cm}^{2}$,

Table 2 GBB-24 scores and quartile values (means \pm S.D.) in female patients with postsurgical hypoparathyroidism $(n=25)$ and in sex- and age-matched controls $(n=25)$.

\begin{tabular}{llccr}
\hline Scales & Score & Patients & Controls & $\boldsymbol{P}$-value \\
\hline Exhaustion tendency & Score & $8.9 \pm 5.6$ & $5.6 \pm 4.9$ & 0.027 \\
Gastric symptoms & Quartile value & $3.1 \pm 1.1$ & $2.3 \pm 1.3$ & 0.069 \\
& Score & $4.2 \pm 3.6$ & $2.6 \pm 2.7$ & 0.084 \\
Pain in the limbs & Quartile value & $3.2 \pm 1.1$ & $2.5 \pm 1.3$ & 0.066 \\
& Score & $10.1 \pm 6.3$ & $6.9 \pm 4.4$ & 0.039 \\
Heart complaints & Quartile value & $3.1 \pm 1.0$ & $2.6 \pm 1.2$ & 0.097 \\
Global socre of discomfort (GSD) & Score & $6.8 \pm 5.2$ & $3.8 \pm 3.1$ & 0.021 \\
& Quartile value & $3.2 \pm 0.8$ & $2.7 \pm 1.0$ & 0.048 \\
& Score & $30.0 \pm 17.5$ & $18.9 \pm 11.6$ & 0.011 \\
& Quartile value & $3.1 \pm 1.0$ & $2.5 \pm 1.1$ & 0.036 \\
\hline
\end{tabular}


z-score $1.11 \pm 1.14, P=0.0005)$ and at the hip (BMD femoral neck $0.96 \pm 0.16 \mathrm{~g} / \mathrm{cm}^{2}$, z-score $0.69 \pm 1.13$, $P=0.015 ; \quad$ BMD trochanter $0.85 \pm 0.10 \mathrm{~g} / \mathrm{cm}^{2}$, Z-score $0.88 \pm 0.65, P<0.0001)$. Z-scores of radius $\mathrm{BMD}$ did not differ significantly from the age-matched expected mean values of the reference database (total radius $-0.21 \pm 0.88, P=0.32 ;$ trabecular radius $-0.27 \pm 1.06, P=0.28)$.

\section{Discussion}

Our study gives compelling evidence that patients with chronic hypoparathyroidism receiving standard treatment with calcium and vitamin D suffer from a significant impairment of well-being and mood. The global scores of different psychometric questionnaires exceeded by one standard deviation the means of the respective normal ranges and the scores found in the matched controls. Furthermore, subscale analysis revealed a characteristic pattern of mood alterations with predominantly high scores for anxiety, phobic anxiety and their physical equivalents (e.g. heart complaints/palpitations). The need for chronic intake of replacement therapy as such cannot explain the mood impairment observed in the hypoparathyroid patients, as more complex replacement regimens in other endocrinopathies are not associated with a similar impairment of well-being (19).

Patients and controls did not differ concerning sex, age, time since thyroid surgery, menopausal status or frequency of estrogen/progestin and thyroxine replacement therapy. We cannot exclude that variations in magnesium levels or intake were a confounding variable for our findings, as only 7 of 25 patients regularly took magnesium replacement. As both patients and controls had previously undergone neck surgery, we can exclude influences of the surgical procedure itself on well-being. However, the postoperative experience of tetanic episodes may have triggered changes in well-being. Although we cannot fully exclude this possibility, it seems unlikely as in this case a gradual improvement in well-being with time after surgery should be expected, which was not observed. It is unlikely that our findings are influenced by a selection bias, as we included all patients responding to our initial mail contact, i.e. $80 \%$ of the total unselected outpatient pool. However, patients seen in a secondary/ tertiary center like ours might be more 'difficult' than hypoparathyroid patients routinely treated by a general practitioner. However, such a bias is unlikely to explain the observed extent of impairment and the characteristic pattern of alterations in mood.

In patients with long-standing hypoparathyroidism (i.e. duration of disease longer than 8-10 years) basal ganglia or even diffuse brain calcification may occur (20-23). While this condition is generally asymptomatic, in some cases an association with cognitive dysfunction (23) and even with organic mood disorder $(24,25)$ has been reported. We did not perform computerized tomography scans of the brain in our patients. However, it is not likely that the observed mood impairment can be explained by cerebral calcifications, as only 5 of the 25 patients had a duration of disease longer than 8 years. Moreover, regression analysis did not reveal any correlation between duration of disease and well-being or mood scores. Thus, the observed mood alterations are more likely due to functional rather than structural changes. However, serum calcium did not significantly correlate with mood scale scores in the hypoparathyroid patients.

It is conceivable that the impairment of well-being results directly from PTH deficiency not involving changes in calcium homeostasis. PTH specifically binds to the recently discovered $\mathrm{PTH} 2$ receptor, which is expressed in several brain regions (26). However, recently a brain-specific ligand to the PTH2 receptor, a hypothalamic neuropeptide, has been described (27). Also the expression of 25-hydroxyvitamin D-1 alpha-hydroxylase, a target enzyme of PTH action in the kidney, has been recently demonstrated in brain tissue (28). However, its physiological role in the central nervous system remains to be determined.

The majority of the hypoparathyroid patients presented with serum calcium levels in the accepted therapeutic target range; thus they could be considered as adequately treated. However, as compared with the controls, mean serum calcium was significantly lower while mean serum phosphorus was significantly higher in the patients with hypoparathyroidism. There was a positive correlation between serum and urinary calcium in both our patients and controls; however, in comparison with controls the regression line of the patient group showed an upward shift. This reflects the reduced tubular calcium reabsorption in hypoparathyroidism (29) leading to hypercalciuria even in the presence of normal serum calcium levels (10). The simultaneously decreased tubular phosphorus secretion is reflected by the negative correlation between serum calcium and serum phosphorus seen in the patients, but not in the controls. Thus, our results confirm that calcium and vitamin D treatment is unable to restore a physiological calcium/phosphorus homeostasis putting hypoparathyroid patients at a higher risk of extra-osseous calcification. The patients from our study group diagnosed with nephrolithiasis or cataracts further illustrate this.

In accord with previous studies in smaller numbers of patients (30-32) we found normal to increased BMD at all sites of measurement in the hypoparathyroid patients. Similar to the results of Duan et al. (33) in a longitudinal study with ten patients, BMD in our patients was higher at the lumbar spine than at the distal radius. Langdahl et al. (34) found a reduction in bone resorption rate and activation frequency and a prolonged quiescent period in bone biopsy specimens 
from 12 hypoparathyroid patients, thereby giving further evidence to the notion that vitamin $\mathrm{D}$ alone is not able to normalize bone turnover.

In conclusion, we have shown that patients with PSHP receiving calcium and vitamin D standard treatment exhibit not only the known dysregulation of calcium/phosphorus homeostasis but do also suffer from a clinically relevant major impairment of mood with a pattern usually seen in anxiety disorders. Thus more physiological treatment options for hypoparathyroidism including their impact on well-being need to be evaluated. After initial attempts to restore normal PTH secretion in hypoparathyroidism by parathyroid allotransplantation $(35,36)$, during recent years important progress has been made in this field now opening up the perspective of parathyroid allotransplantation without the requirement of immunosuppression (3739). Moreover, it has been shown recently that s.c. injected PTH(1-34) restores the physiological calcium homeostasis in patients with hypoparathyroidism (40). As PTH may soon become available as a new treatment option for osteoporosis, the time for its evaluation in the treatment of chronic hypoparathyroidism may have come.

\section{References}

1 al-Suliman NN, Ryttov NF, Qvist N, Blichert-Toft M \& Graversen HP. Experience in a specialist thyroid surgery unit: a demographic study, surgical complications, and outcome. European Journal of Surgery $1997 \mathbf{1 6 3} 13-20$.

2 Pappalardo G, Guadalaxara A, Frattaroli FM, Illomei G \& Falaschi P. Total compared with subtotal thyroidectomy in benign nodular disease: personal series and review of published reports. European Journal of Surgery $1998 \mathbf{1 6 4} 501-506$.

3 Rimpl I \& Wahl RA. Surgery of nodular goiter: postoperative hypocalcemia in relation to extent of resection and manipulation of the parathyroid glands. Langenbecks Archiv der Chirurgie Supplement Kongressband $1998 \mathbf{1 1 5}$ 1063-1066.

4 Thomusch O, Machens A, Sekulla C, Ukkat J, Kippert H, Gastinger I et al. Multivariate analysis of risk factors for postoperative complications in benign goiter surgery: prospective multicenter study in Germany. World Journal of Surgery 200024 324-329.

5 Riehl J, Kierdorf H, Schmitt H, Suiter T \& Sieberth HG. Prevalence of goiter in the Aachen area. Ultrasound volumetry of the thyroid gland of 1336 adults in an endemic goiter region. Ultraschall in der Medizin $19951684-89$.

6 Avioli LV. The therapeutic approach to hypoparathyroidism. American Journal of Medicine 197457 34-42.

7 Haussler MR \& Cordy PE. Metabolites and analogues of vitamin D. Which for what? Journal of the American Medical Association $1982247841-844$.

8 Rizzoli R \& Bonjour JP. Vitamin D treatment of hypoparathyroid patients. European Journal of Endocrinology 1997136 25-27.

9 Schilling T \& Ziegler R. Current therapy of hypoparathyroidism a survey of German endocrinology centers. Experimental and Clinical Endocrinology and Diabetes 1997105 237-241.

10 Nordin BE \& Peacock M. Role of kidney in regulation of plasma calcium. Lancet 19692 1280-1283.

11 Derogatis LR, Lipman RS \& Covi L. SCL-90: an outpatient psychiatric rating scale - preliminary report. Psychopharmacology Bulletin $1973913-28$.
12 Franke GH. SCL-90-R. Die Symptom-Checkliste von Derogatis Deutsche Version - Manual. Goettingen: Beltz Test GmbH, 1995.

13 Zerssen D \& Koeller DM. Die Beschwerden-Liste (Manual). Klinische Selbstbeurteilungsskalen aus dem Muenchner Psychiatrischen Informationssystem (PSYCHIS). Weinheim: Beltz-Verlag, 1976.

14 Braehler E \& Scheer JW. Skalierung psychosomatischer Beschwerdenkomplexe mit dem Giessener Beschwerdebogen (GBB). Psychotherapie und Medizinische Psychologie 197929 14-27.

15 Braehler E, Surrey HW \& Scheer JW. Der Giessener Beschwerdebogen (GBB-Tabellenband). Giessen: Institut fuer Medizinische Psychologie, 1983.

16 Dequeker J, Pearson J, Reeve J, Henley M, Bright J, Felsenberg D et al. Dual X-ray absorptiometry - cross-calibration and normative reference ranges for the spine: results of a European Community Concerted Action. Bone 199517 247-254.

17 Pearson J, Dequeker J, Reeve J, Felsenberg D, Henley M, Bright J et al. Dual X-ray absorptiometry of the proximal femur: normal European values standardized with the European Spine Phantom. Journal of Bone and Mineral Research $199510315-324$.

18 Schneider P, Butz S, Allolio B, Borner W, Klein K, Lehmann R et al. Multicenter German reference data base for peripheral quantitative computer tomography. Technology and Health Care 19953 $69-73$.

19 Arlt W, Callies F, van Vlijmen JC, Koehler I, Reincke M, Bidlingmaier $\mathrm{M}$ et al. Dehydroepiandrosterone replacement in women with adrenal insufficiency. New England Journal of Medicine 1999 341 1013-1020.

20 Posen S, Clifton-Bligh P \& Cromer T. Computerized tomography of the brain in surgical hypoparathyroidism. Annals of Internal Medicine $197991415-417$.

21 Forman MB, Sandler MP, Danziger A \& Kalk WJ. Basal ganglia calcification in postoperative hypoparathyroidism. Clinical Endocrinology 198012 385-390.

22 Illum F \& Dupont E. Prevalences of CT-detected calcification in the basal ganglia in idiopathic hypoparathyroidism and pseudohypoparathyroidism. Neuroradiology 198527 32-37.

23 Kowdley KV, Coull BM \& Orwoll ES. Cognitive impairment and intracranial calcification in chronic hypoparathyroidism. American Journal of Medical Sciences 1999317 273-277.

24 Trautner RJ, Cummings JL, Read SL \& Benson DF. Idiopathic basal ganglia calcification and organic mood disorder. American Journal of Psychiatry $1988 \mathbf{1 4 5}$ 350-353.

25 Lopez-Villegas D, Kulisevsky J, Deus J, Junque C, Pujol J, Guardia E et al. Neuropsychological alterations in patients with computed tomography-detected basal ganglia calcification. Archives of Neurology 199653 251-256.

26 Usdin TB, Gruber C \& Bonner TI. Identification and functional expression of a receptor selectively recognizing parathyroid hormone, the PTH2 receptor. Journal of Biological Chemistry 1995 270 15455-15458.

27 Usdin TB, Hoare SR, Wang T, Mezey E \& Kowalak JA. TIP39: a new neuropeptide and PTH2-receptor agonist from hypothalamus. Nature Neuroscience $19992941-943$.

28 Fu GK, Lin D, Zhang MY, Bikle DD, Shackleton CH, Miller WL et al. Cloning of human 25-hydroxyvitamin D-1 alpha-hydroxylase and mutations causing vitamin D-dependent rickets type 1. Molecular Endocrinology 199711 1961-1970.

29 Mortensen L, Hyldstrup L \& Charles P. Effect of vitamin D treatment in hypoparathyroid patients: a study on calcium, phosphate and magnesium homeostasis. European Journal of Endocrinology 1997136 52-60.

30 Seeman E, Wahner HW, Offord KP, Kumar R, Johnson WJ \& Riggs BL. Differential effects of endocrine dysfunction on the axial and the appendicular skeleton. Journal of Clinical Investigation 1982 $691302-1309$.

31 Abugassa S, Nordenstrom J, Eriksson S \& Sjoden G. Bone mineral density in patients with chronic hypoparathyroidism. Journal of Clinical Endocrinology and Metabolism 199376 1617-1621.

32 Fujiyama K, Kiriyama T, Ito M, Nakata K, Yamashita S, Yokoyama $\mathrm{N}$ et al. Attenuation of postmenopausal high turnover 
bone loss in patients with hypoparathyroidism. Journal of Clinical Endocrinology and Metabolism $1995 \mathbf{8 0} 2135-2138$.

33 Duan Y, De Luca V \& Seeman E. Parathyroid hormone deficiency and excess: similar effects on trabecular bone but differing effects on cortical bone. Journal of Clinical Endocrinology and Metabolism $199984718-722$.

34 Langdahl BL, Mortensen L, Vesterby A, Eriksen EF \& Charles P. Bone histomorphometry in hypoparathyroid patients treated with vitamin D. Bone 199618 103-108.

35 Duarte B, Mozes MF, John E, Aronson I, Pollak R \& Jonasson O. Parathyroid allotransplantation in the treatment of complicated idiopathic primary hypoparathyroidism. Surgery $1985 \mathbf{9 8}$ 1072-1076.

36 Zeng $\mathrm{Q}$. Allotransplantation of parathyroid glands to treat intractable hypoparathyroidism. Surgery 198699 131-132.

$37 \mathrm{Fu} \mathrm{XW} \mathrm{\&} \mathrm{Sun} \mathrm{AM.} \mathrm{Microencapsulated} \mathrm{parathyroid} \mathrm{cells} \mathrm{as} \mathrm{a}$ bioartificial parathyroid. In vivo studies. Transplantation $1989 \mathbf{4 7}$ $432-435$.
38 Tolloczko T, Wozniewicz B, Gorski A, Sawicki A, Narot I, Migaj M et al. Cultured parathyroid cells allotransplantation without immunosuppression for treatment of intractable hypoparathyroidism. Annals of Transplantation 19961 51-53.

39 Hasse C, Klock G, Schlosser A, Zimmermann U \& Rothmund M. Parathyroid allotransplantation without immunosuppression. Lancet 1997350 1296-1297.

40 Winer KK, Yanovski JA \& Cutler GB Jr. Synthetic human parathyroid hormone 1-34 vs calcitriol and calcium in the treatment of hypoparathyroidism. Journal of the American Medical Association 1996276 631-636.

Received 11 April 2001

Accepted 15 October 2001 